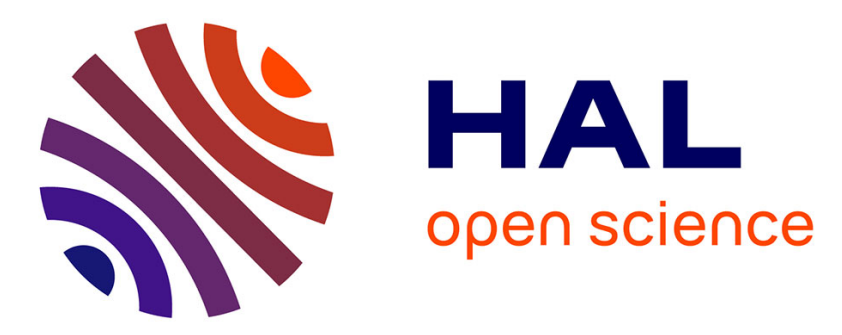

\title{
A prediction-based optimal gain selection in RISE feedback control for hard disk drive
}

Manel Taktak-Meziou, Ahmed Chemori, Jawhar Ghommam, Nabil Derbel

\section{To cite this version:}

Manel Taktak-Meziou, Ahmed Chemori, Jawhar Ghommam, Nabil Derbel. A prediction-based optimal gain selection in RISE feedback control for hard disk drive. CCA: Conference on Control Applications, Oct 2014, Juan Les Antibes, France. pp.2114-2119, 10.1109/CCA.2014.6981615 . lirmm01723921

\section{HAL Id: lirmm-01723921 https://hal-lirmm.ccsd.cnrs.fr/lirmm-01723921}

Submitted on 5 Mar 2018

HAL is a multi-disciplinary open access archive for the deposit and dissemination of scientific research documents, whether they are published or not. The documents may come from teaching and research institutions in France or abroad, or from public or private research centers.
L'archive ouverte pluridisciplinaire HAL, est destinée au dépôt et à la diffusion de documents scientifiques de niveau recherche, publiés ou non, émanant des établissements d'enseignement et de recherche français ou étrangers, des laboratoires publics ou privés. 


\title{
A Prediction-Based Optimal Gain Selection in RISE Feedback Control for Hard Disk Drive
}

\author{
M. Taktak-Meziou ${ }^{1}$, A. Chemori ${ }^{2}$, J. Ghommam $^{1}$, and N. Derbel ${ }^{1}$
}

\begin{abstract}
This paper presents a prediction-based optimal gain selection in Robust Integral Sign of the Error (RISE) based Neural Network (NN) approach. Previous research has shown that combining a feedforward term with a feedback control element yields an asymptotically stable closed-loop system. The proposed approach adds a prediction-based optimal technique which minimizes a quadratic performance index to calculate an optimal feedback gain. The resulting novel controller, called P-RISE-NN, is applied for a track following problem of a HardDisc-Drive servo-system. Simulation studies are used to show the efficiency of the proposed control scheme and its robustness against external disturbances and parametric uncertainties in the system. The authors believe that the proposed control solution combining RISE with a predictive control approach has never been conducted before.
\end{abstract}

\section{INTRODUCTION}

In the real world, the majority of systems have nonlinear dynamic behaviors. Their sensitivity to external environmental effects can be reflected through the existence of uncertainties and poor knowledge of the parameters in the system's dynamics. To deal with these issues, several solutions have been investigated in the literature and many control approaches have been proposed. Their common goal is to design an effective controller that is able to compensate the actual disturbances and to minimize the effects of nonlinearities. These factors, if not well compensated, can generate significant errors and degrade the overall system performance.

Recently, a new controller named Robust Integral of the Sign of the Error (RISE) has been proposed [1]. The major outcome of this control scheme is that the Asymptotic Stability (AS) of the considered nonlinear class of systems is ensured. In Ref. [2], the RISE control method has been used to solve a tracking problem by compensating additive system disturbances with bounded derivatives. A combination of RISE method and a high gain observer has been developed in [3]. Such a technique provides a Semi Global Uniformly Ultimately Bounded (SGUUB) tracking with the compensation of unstructured uncertainties. Moreover, in [4]-[6], the proposed high gain feedback method is applied for identification of some perspective systems, such as paracatadioptric vision and friction function.

\footnotetext{
${ }^{1}$ M. Taktak-Meziou, J. Ghommam, and N. derbel are with the CEM Lab, University of Sfax, Department of Electrical Engineering, National School of Engineers of Sfax (ENIS), BP, W 3038 Sfax Tunisia. manel.taktak@yahoo.com, jawhar.ghommamegmail.com, n.derbeldenis.rnu.tn

${ }^{2} \mathrm{~A}$. Chemori is with the LIRMM, University Montpellier 2 / CNRS, UMR5506, CC477, 161 rue Ada, 34095 Montpellier Cedex 5, France. Ahmed.Chemori@lirmm. fr
}

Many researchers have investigated methods of reducing the control effort and improving the steady state response of the RISE feedback controller. The key idea is to combine the high gain controller RISE with a feedforward control term. The universal approximation property of Neural Networks (NN) is suited for enhancing the system performance and dealing with lack of knowledge of the system dynamics. Such combination, as developed in [7], guarantees the Asymptotic Stability (AS) of the resulting closed-loop system as well as the boundedness of the $\mathrm{NN}$ weights while achieving the tracking task.

The main objective of this paper is to integrate a predictive approach with the RISE based Neural Network (RISE-NN) structure. The basic idea was inspired from the sensitivity of the controlled system behavior in the presence of small variations of the RISE control gain parameter. This novel control approach will be called Prediction-based RISE-NN controller (P-RISE-NN) throughout the paper. The prediction-based approach developed in this paper is not used to calculate an optimal control sequence, but to compute an optimal control gain. We propose the application of the new approach $\mathrm{P}$ RISE-NN on a Hard-Disc-Drive (HDD) servo-system, as has never been done before. Various numerical simulations in different operating conditions will be presented to show the efficiency of the proposed control scheme. The investigated scenarios deal with the tracking problem in presence of external disturbances as well as parametric uncertainties in the system dynamics.

This paper is organized as follows: The second section, the HDD system description and control objectives are presented. The third section is dedicated to a background on the RISE based NN approach. The fourth section contains details of the proposed prediction-based optimal gain tuning. Numerical simulations are presented and discussed in the fifth section to show the effectiveness of the proposed control method. The paper ends with concluding remarks.

\section{SYSTEM DESCRIPTION AND CONTROL PROBLEM FORMULATION}

With the significant progress in Hard-Disc-Drives (HDD) technology, different research communities have shown interest in this field. Therefore, several solutions have been proposed to meet the increasing demands for higher operating performance. Fig. 1 is an overview of a typical HDD with a Voice-Coil-Motor (VCM) actuator.

The Read/Write (R/W) head, which is mounted on the actuator tip, is the most important component. It is supported by a pivot arm ensuring its displacement from one track to 


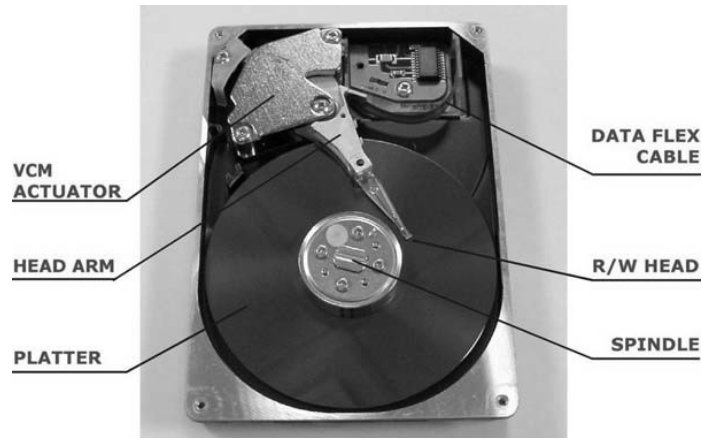

Fig. 1: View of a typical HDD system and its main components [8]

another to Read/Write data on/from a desired track. As given in [9], the HDD servo-positioning system can be modeled as follows:

$$
\begin{aligned}
M(q) \ddot{q}+F(q, \dot{q}) & =u \\
y & =q
\end{aligned}
$$

where $M(q)$ denotes the system inertia $(M(q)>0) . y, \dot{y}$ and $\ddot{y}$ are respectively the position, the velocity and the acceleration of the VCM-actuator/head tip. $u$ is the control input. $F(q, \dot{q})$ is a nonlinear function representing the pivot bearing hysteresis friction. The dynamics of $F(q, \dot{q})$ are represented through the LuGre friction model which covers all the static and dynamic characteristics of hysteresis friction. It can be expressed as follows:

$$
\begin{aligned}
F(q, \dot{q}) & =\sigma_{0} z+\sigma_{1} \dot{z}+\sigma_{2} \dot{q} \\
\dot{z} & =\dot{q}-\alpha(\dot{q})|\dot{q}| z \\
\alpha(\dot{q}) & =\frac{\sigma_{0}}{f_{c}+\left(f_{s}-f_{c}\right) e^{-\left(\frac{\dot{q}}{q_{s}}\right)^{2}}}
\end{aligned}
$$

where $z$ is an immeasurable internal state of the friction model. $\sigma_{0}, \sigma_{1}$, and $\sigma_{2}$ are the hysteresis model parameters representing the stiffness of bristles, the micro damping coefficient and viscous coefficient respectively. $f_{s}$ is the static force, $f_{c}$ is the Coulomb friction force and $q_{s}$ is the Stribeck velocity parameter [10]. These above parameters are chosen as $M(q)=1, \sigma_{0}=10^{5}, \sigma_{1}=\sqrt{10^{5}}, \sigma_{2}=0.4$, $f_{s}=1.5, f_{c}=1$, and $\dot{q}_{s}=10^{-3}$.

Two main control problems can be distinguished for the HDD servo-positioning-system namely track seeking and track following control. In the former, the controller aims to steer rapidly the head on the target track with a minimum control effort. However, in the latter, the controller has to maintain the head as close as possible to the center of the target track.

Many sources of errors can be found in the HDD servosystem such as input disturbances caused by external shocks and vibrations, inaccuracies generated by the head movement effects, output disturbances induced by the spindle motor rotation effects and measurement noise.

The main objective of the present work can be formulated as follows: "Design a control law $u(t)$ which is able to ensure the displacement of the $\mathrm{R} / \mathrm{W}$ head from one desired track to another in such a way that the head tip is maintained as close as possible to the target track center while treating data. Speed, accuracy and robustness against external disturbances and parameter's uncertainties have to be ensured such as, for a given target reference $q_{d}(t)$ :

$$
\lim _{t \rightarrow \infty}\left|q_{d}(t)-q(t)\right|=\lim _{t \rightarrow \infty}\left|e_{1}(t)\right|=0
$$

where $q(t)$ is the measured position of the HDD head tip and $e_{1}$ is the tracking position error."

Assumption 1: The desired Trajectory $q_{d}(t)$ and its two first derivatives $q_{d}(t), \dot{q}_{d}(t)$, and $\ddot{q}_{d}(t)$ exist and are bounded. In order to facilitate the subsequential analysis, filtered tracking errors $e_{2}(t)$ and $r(t)$ are introduced, they are defined as follows:

$$
\begin{aligned}
e_{2} & =\dot{e}_{1}+\alpha_{1} e_{1} \\
r & =\dot{e}_{2}+\alpha_{2} e_{2}
\end{aligned}
$$

where $\alpha_{1}$ and $\alpha_{2}$ denote some positive constants.

Remark 1: $r(t)$ is an immeasurable error since its expression (8) depends on $\ddot{q}(t)$.

\section{BACKGROUND ON RISE-NN CONTROL}

In this section, a brief background on the RISE feedback based Neural Network (RISE-NN) control is presented. First, the universal approximation property of $\mathrm{NN}$ is introduced to develop a feedforward controller. Then, the RISE feedback principle is described for the system (1)-(2).

\section{A. Universal approximation of NNs: Feedforward control}

Consider $\mathbb{S}$ to be a compact set and $f(x)$ a function defined as $f: \mathbb{S} \rightarrow \mathbb{R}^{a+1}$. A three-layer $\mathrm{NN}$ is chosen in this paper to estimate $f(x)$ such that:

$$
f(x)=W^{\top} \sigma\left(V^{\top} x\right)+\varepsilon(x)
$$

were $x(t) \in \mathbb{R}^{a+1}$ is the inputs vector, $V \in \mathbb{R}^{(a+1) \times L}$ is a matrix of bounded constant weights for the first-to-second layers and $W \in \mathbb{R}^{(L+1) \times 1}$ are the ideal weights for the secondto-third layers. $a$ is the number of neurons in the input layer. $L$ is the number of neurons in the hidden layer. The third layer, the output layer, is assumed with only one neuron. $\sigma(.) \in \mathbb{R}^{L+1}$ is the activation function and $\varepsilon(x) \in \mathbb{R}$ is the functional error approximation.

Remark 2: In this paper, the activation function $\sigma($.$) is a$ radial basis function taking the following general form

$$
\sigma\left(x_{i}\right)=\exp \left(-\frac{\left\|x_{i}-c_{i}\right\|^{2}}{\sigma_{i}^{2}}\right), \quad \forall i \in \mathbb{R}
$$

where $c_{i}$ is the center of the basis function and $\sigma_{i}$ is its width. Both are chosen a prior and kept unchanged for each basis function throughout this work.

Based on the universal approximation property of NNs [11], the function $f(x)$, as given in (9), can be estimated as follows:

$$
\hat{f}(x)=\hat{W}^{\top} \sigma\left(V^{\top} x\right)
$$


were $\hat{W}^{\top} \in \mathbb{R}^{(L+1) \times 1}$ is the estimate of the ideal weight matrix.

Assumption 2: The boundedness of the ideal weights and the activation function are assumed to be ensured so that $\|W\| \leq W_{m},\|\sigma\| \leq \sigma_{m}$, where $W_{m}$ and $\sigma_{m}$ are some positive known constants.

\section{B. RISE feedback controller}

In order to develop the control input $u(t)$, both sides of (8) are multiplied by $M(q)$. Then, using (1)-(2), we obtain:

$$
M(q) r=F_{d}+S-u
$$

where $F_{d}$ and $S$ are auxiliary functions defined as follows:

$$
\begin{aligned}
F_{d} & =M\left(q_{d}\right) \ddot{q}_{d}+F\left(q_{d}, \dot{q}_{d}\right) \\
S & =M(q)\left(\alpha_{1} \dot{e}_{1}+\alpha_{2} \dot{e}_{2}\right)+F(q, \dot{q})-F\left(q_{d}, \dot{q}_{d}\right)
\end{aligned}
$$

Based on the estimation (11), $F_{d}$ can be approximated as:

$$
F_{d}=W^{\top} \sigma\left(V^{\top} x_{d}\right)+\varepsilon\left(x_{d}\right)
$$

where $x_{d}$ is the input vector defined as: $x_{d}=$ $\left[\begin{array}{llll}1, & q_{d}, & \dot{q}_{d}, & \ddot{q}_{d}\end{array}\right]^{\top}$. Based on assumption $1, \varepsilon\left(x_{d}\right)$ verifies the following boundary conditions:

$$
\begin{aligned}
&\left\|\varepsilon\left(x_{d}\right)\right\| \leq \varepsilon_{N} \\
&\left\|\dot{\varepsilon}\left(x_{d}\right)\right\| \leq \varepsilon_{N}^{\prime}
\end{aligned}
$$

where $\varepsilon_{N}$ and $\varepsilon_{N}^{\prime}$ are known positive constants.

Then, as proposed in [1], we define the RISE feedback control term as:

$$
\begin{aligned}
\mu(t) & =\left(k_{s}^{o p t}+1\right) e_{2}(t)-\left(k_{s}^{o p t}+1\right) e_{2}(0) \\
& +\int_{0}^{t}\left[\left(k_{s}^{o p t}+1\right) \alpha_{2} e_{2}(s)+\beta_{1} \operatorname{sign}\left(e_{2}(s)\right)\right] d s
\end{aligned}
$$

where $k_{s}^{\text {opt }}, \beta_{1} \in \mathbb{R}^{+}$are positive constant feedback gains.

Remark 3: $k_{s}^{\text {opt }}$ is a non-varying feedback gain in the classical RISE controller as proposed in [1]. The key idea of this paper, which is detailed in the next section, is to use a prediction-based optimal technique to tune this parameter for the best tracking performance of a HDD servo-positioning system.

The first time derivative of equation (18) gives:

$$
\dot{\mu}(t)=\left(k_{s}^{o p t}+1\right) r(t)+\beta_{1} \operatorname{sgn}\left(e_{2}(t)\right)
$$

The control input $u(t)$ of the system (1)-(2) is composed of two terms, the RISE feedback term plus the feedforward NN estimation term, that is:

$$
u=\mu+\hat{F}_{d}
$$

were $\hat{F}_{d}$ is the estimation obtained through (11) and the estimates of the NN weights $\hat{W}^{\top}$ are generated online through the following expression

$$
\dot{\hat{W}}=K\left[\sigma\left(V^{\top} x_{d}\right) e_{2}^{\top}-\kappa \hat{W}\right]
$$

with $\kappa$ is a positive constant design parameter. $K=K^{\top}>0$ is a constant positive definite gain matrix.
Remark 4: Since the RISE-NN control structure developed in this paper is a special case of [12] with a fixed hidden layer weights, and by assumption 2 , the boundedness of $\dot{\hat{W}}$ can be proven. In fact, we address an optimization problem to find $K_{S}^{o p t}$ under the constraint that $k_{s}^{o p t}>0$. The stability analysis of [12] has been performed for a positive feedback gain $k_{s}^{o p t}$. Therefore, the boundedness of the NN weights is guaranteed.

Consider equations (11) and (15), the time derivative of $u(t)$ can be expressed as:

$$
\dot{u}=\frac{d}{d t}\left(\hat{W}^{\top} \sigma\left(V^{\top} x_{d}\right)\right)+\left(k_{s}^{o p t}+1\right) r(t)+\beta_{1} \operatorname{sgn}\left(e_{2}(t)\right)
$$

Using (12) and (22) to formulate the closed-loop system dynamics, we obtain:

$$
\begin{aligned}
M(q) \dot{r}= & -\dot{M}(q) r+\dot{F}_{d}+\dot{S}-\dot{u} \\
= & -\frac{1}{2} \dot{M}(q) r+\tilde{W}^{\top} \sigma\left(V^{\top} x_{d}\right)+\varepsilon\left(x_{d}\right)-\left(k_{s}^{o p t}+1\right) r(t) \\
& +\left(-\frac{1}{2} \dot{M}(q) r+\dot{S}+e_{2}\right)-\beta_{1} \operatorname{sgn}\left(e_{2}(t)\right)-e_{2}
\end{aligned}
$$

where $\tilde{W}^{\top}=W^{\top}-\hat{W}^{\top}$ is the weights estimation error.

Consider now:

$$
\begin{aligned}
\tilde{N} & =-\frac{1}{2} \dot{M}(q) r+\dot{S}+e_{2} \\
N_{B_{1}} & =\varepsilon\left(x_{d}\right) \\
N_{B_{2}} & =\tilde{W}^{\top} \sigma\left(V^{\top} x_{d}\right)
\end{aligned}
$$

then equation (23) can be expressed as follows:

$$
\begin{aligned}
M(q) \dot{r}= & -\frac{1}{2} \dot{M}(q) r+\tilde{N}+N_{B_{1}}+N_{B_{2}}-e_{2} \\
& -\left(k_{s}^{o p t}+1\right) r(t)-\beta_{1} \operatorname{sgn}\left(e_{2}(t)\right)
\end{aligned}
$$

Thanks to the mean value theorem, $\tilde{N}$ can be upper bounded as follows:

$$
\|\tilde{N}\|=\left\|-\frac{1}{2} \dot{M}(q) r+\dot{S}+e_{2}\right\| \leq \rho(\|z\|)\|z\|
$$

where $z(t)=\left[\begin{array}{lll}e_{1} & e_{2} & r\end{array}\right]^{\top} \in \mathbb{R}^{3}$ and $\rho(\|z\|)$ is a positive non decreasing bounding function.

\section{MAIN CONTRIBUTION: THE PROPOSED P-RISE-NN CONTROL}

In the standard RISE-NN, choosing the appropriate value of $k_{s}^{o p t}$ in (18) is made off line and kept constant. However, since the closed-loop system behavior is very sensitive to small variations of this feedback gain, we propose to tune it using a prediction based optimization technique.

In this section, the proposed prediction-based optimal gain tuning approach is described. Fig. 2 illustrates the block diagram of the proposed P-RISE-NN control scheme.

The tuning basic idea is inspired from the Model Predictive Control (MPC) principle [13]. In the proposed scheme, the solution is not an optimal control sequence, but rather an optimal feedback gain parameter $k_{s}^{\text {opt }}$.

The control algorithm consists of the following four steps with illustration in Fig.3: 


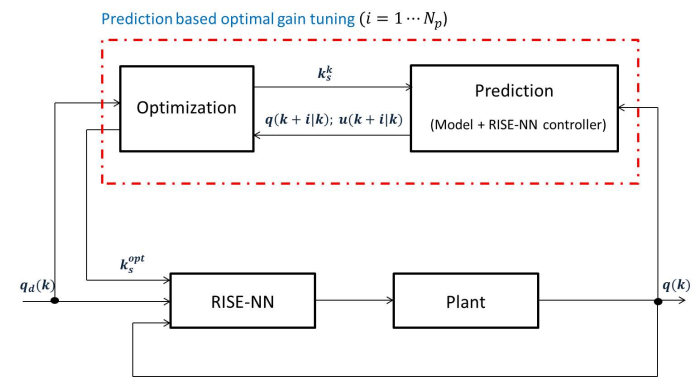

Fig. 2: Block Diagram of the P-RISE-NN control scheme

- Step 1: At each sample time $k=n T_{e}$, were $n$ is a positive integer and $T_{e}$ is the sample time, the future outputs of the plant are predicted over a predefined prediction horizon $N_{p}$. The predictions $\hat{q}(k+i \mid k)$ and $\hat{u}(k+i \mid k)$ for $i=1, \ldots, N_{p}$ are determined using the model of the system described by (1)-(2) and the classical RISE-NN procedure detailed in section III-B.

- Step 2: The optimal control gain $k_{s}^{\text {opt } \mid k}$ at time instant $k$, is determined through the minimization of a performance index. This latter is chosen to be a quadratic function including the future control inputs and the future tracking errors defined by:

$$
e(k+i \mid k)=q_{d}(k+i \mid k)-\hat{q}(k+i \mid k)
$$

where $q_{d}(k+i \mid k)$ is the reference sequence to follow assumed to be known as a priori. $\hat{q}(k+i \mid k)$ is the predicted output sequence of the plant at time instant $k$. Accordingly, the cost function $J$ is defined as follows:

$$
J=\sum_{i=1}^{N_{p}}\|e(k+i \mid k)\|_{Q}^{2}+\|\hat{u}(k+i \mid k)\|_{R}^{2}
$$

where $\|\mathbf{x}\|_{M}^{2}=\mathbf{x}^{T} M \mathbf{x} . Q \geq 0$ and $R \geq 0$ are symmetric positive definite weighting matrices.

The main objective of the prediction-based optimal technique is to compute the best value of the feedback gain $k_{s}^{\text {opt } \mid k}$ that minimizes the performance index $J$, that is:

$$
k_{s}^{o p t \mid k} \equiv A r g \min _{k_{s}} J
$$

subject to $k_{s}^{o p t}>0$.

The performed optimization is in general a non convex problem because of the high nonlinearities in the system dynamics. Consequently, a numerical nonlinear programming is crucial to compute the optimal solution.

- Step 3: The computed optimal solution is used in the controller over the next sample period $[k, k+1]$.

- Step 4: The horizon is shifted and the procedure is repeated at the next sampling time.

Fig. 3 illustrates the basic principle of the prediction-based optimal tuning.

Remark 5: Since the HDD model (1)-(2) includes unknown parameters, we propose to approximate these uncertain dynamics as well as to mitigate their effect on the

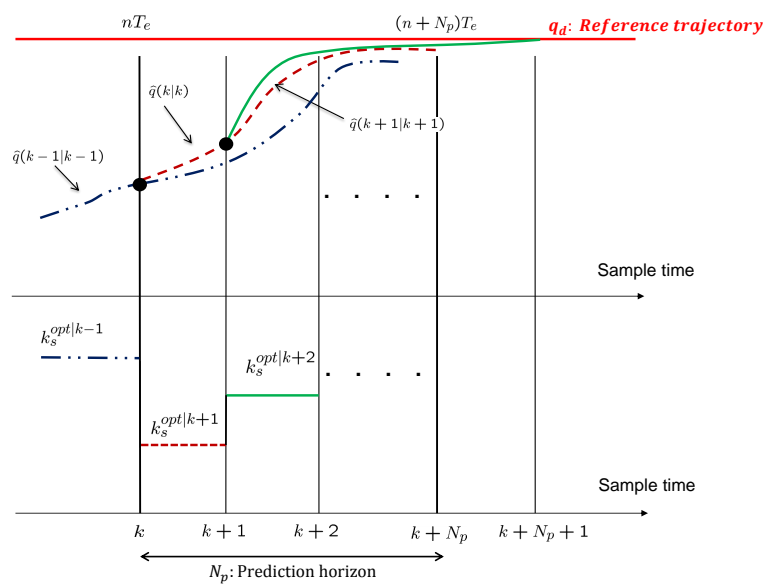

Fig. 3: Prediction-based optimal tuning

controlled system by the mean of NN feedforward control term. Therefore, the prediction model used in the P-RISE$\mathrm{NN}$ procedure is updated at each sample instant by the estimations of NNs.

\section{NUMERICAL SIMULATION RESULTS}

In this section, numerical simulation results of the proposed P-RISE-NN controller are presented and compared to those of a classical RISE-NN controller and a classical Proportional Derivative controller (PD). In simulations, a step reference trajectory varying from 0 to $1 \mu \mathrm{m}$ is considered. The control input (voltage of the actuator) is assumed to be saturated between $-3 v$ and $+3 v$. The parameters of the different controllers are manually tuned to obtain the best closed-loop system's performance. The PD control gains are chosen to be $K_{p}=2 \times 10^{7}$ and $K_{d}=1 \times 10^{3}$. The parameters of the prediction technique in (30) are chosen as: $N_{p}=25$, $Q=100 I$, and $R=100 I$, where $I$ is the identity matrix. The sampling time is $T_{e}=0.05 \mathrm{~ms}$. The system inertia is $m=1$ (normalized value). The RISE control gains in (18) are set to: $\alpha_{1}=\alpha_{2}=1500$ and $\beta_{1}=1$. All the initial conditions are considered at the origin.

Two different scenarios have been performed: The first one deals with the nominal case with external disturbances. In the second scenario, parametric uncertainties of $-40 \%$, $+40 \%$ and $+80 \%$ are considered on the system inertia $M$. The purpose of this scenario is to see whether the controllers are sufficiently robust to compensate parametric changes and maintain the performance of the closed-loop system.

For a comparison purpose between the different proposed control schemes, an energy function is introduced. It is expressed by:

$$
E=\sum_{i=1}^{N_{s i m}}\left|u_{i}\right|
$$

where $N_{s i m}$ is the number of samples in the duration of simulation. $u_{i}$ is the control input value at each sampling time. 


\section{A. Scenario 1: Nominal case and disturbance rejection}

In this scenario, the control feedback gain for the standard RISE-NN controller is set to $k_{s}^{o p t}=1850$. The number of samples is fixed at $N_{\text {sim }}=600$. Several sources of disturbances, which may contribute to the degradation of the overall system performance, are considered in this scenario. We introduce an input disturbance $w_{i n}$, which is often an unknown bounded perturbation satisfying $\left|w_{i n}\right| \leq 3 m V$. For simplicity, we set $w_{\text {in }}=-3 m V$ and assume it to be constant throughout this scenario [8]. Moreover, an unexpected output disturbance $w_{\text {out }}$ is also considered at the time instant $t=$ $15 m s$. $w_{\text {out }}$ is assumed to be an impulse disturbance with an amplitude of $0.3 \mu \mathrm{m}$. In addition, a measurement noise, induced by the position-measurement techniques and/or sensors, is assumed to be a zero-mean Gaussian white noise $\omega_{\text {noise }}$ with a variance $\sigma^{2}=9 \times 10^{-9}(\mathrm{~m})^{2}$.

The obtained simulation results of the closed-loop system are shown in Fig. 4. The following observations can be made:

- The R/W head is able to reach the desired track located at $1 \mu \mathrm{m}$ with all the proposed controllers. However, the best speed and accuracy are obtained with the proposed P-RISE-NN controller as shown in Fig. 4-(a). The PD controller generates large settling time and significant overshoots which degrade the system performance;

- The unexpected disturbance effect at time instant $t=$ $15 m s$ is perceptible in all controllers. Consequently, the $\mathrm{R} / \mathrm{W}$ head is moved away from its current position at a distance of $3 \mu \mathrm{m}$. The RISE-NN and P-RISE-NN approaches have roughly the same disturbance rejection performances. Both generate smaller overshoots and require less control effort (Fig. 4-(b)) compared to PD controller.

- For a comparison purpose, we define the recovery time $t_{r e c}$ as the time needed to reach a tracking error of $2 \%$ of the desired final value from the instant of application of the output disturbance. Both PD and P-RISE-NN are slightly faster than RISE-NN such that the R/W head tip returning to the original desired track is made with shorter recovery time.

For all the proposed controllers, the resulting 5\% settling time, the energy function $E$, the $2 \%$ recovery time $t_{r e c}$ and the generated overshoots are summarized in TABLE I.

TABLE I: Performances of the track following controller in nominal case

\begin{tabular}{|c||c|c|c|}
\hline & PID & RISE-NN & P-RISE-NN \\
\hline Settling time (ms) & 3.15 & 4.5 & 1.2 \\
\hline $\begin{array}{c}\text { Maximum overshoot } \\
\text { (Before disturbance) }\end{array}$ & $40 \%$ & $10 \%$ & absent \\
\hline $\begin{array}{c}\text { Maximum overshoot } \\
\text { (After disturbance) }\end{array}$ & $17 \%$ & $3 \%$ & absent \\
\hline Recovery time (ms) & 2.5 & 3.2 & 2.5 \\
\hline Maximum control input (v) & 3 & 1.1 & 1.3 \\
\hline Energy function $E(\mathrm{v})$ & $2.53 \times 10^{2}$ & 77.31 & 61.08 \\
\hline
\end{tabular}

\section{Computational aspect and practical implementation}

In order to evaluate the average value of the computation time of the proposed P-RISE-NN control scheme, we consider the HDD servo-system operating for 600 steps with the sampling time $T_{e}=0.05 \mathrm{~ms}$. The operating conditions are those of scenario 1 , ie. nominal conditions with disturbance rejection.

The optimization algorithm is based on the predefined function fmincon of Matlab software of MathWorks. The evaluation of the computing time was achieved on a computer equipped with a $1.60 \mathrm{GHz}$ Intel microprocessor.

The computed mean value is given by $t_{m}=10.5 \mathrm{~s}$. The evaluation of the computing time shows that the proposed control approach is not applicable in real-time. Consequently, it becomes interesting to use the programming language $\mathrm{C}^{++}$which is able to improve the computing time by a factor of 10 to 100 .

\section{B. Scenario 2: Robustness towards parameter uncertainties}

In this scenario, uncertainties of $-40 \%,+40 \%$ and $+80 \%$ on the system's inertia parameter $m$ have been considered. The objective is to evaluate the robustness of the three controllers against parametric uncertainties. The obtained simulation results are shown in Fig. 5 and Fig. 6 for PD, RISE-NN and P-RISE-NN controllers respectively.

For uncertainties of $-40 \%$, improvements are observed in the closed-loop system response for all controllers. This can be explained by the fact that the inertial mass is lighter in this case, and a weaker force is exerted on the servo positioning system.

However, for larger uncertainties on the system's inertia parameter, the behavior of the HDD is more affected with PD and classical RISE-NN than the proposed P-RISENN controller. In Fig. 5, it can be clearly seen that with $+40 \%$ and $+80 \%$, the PD generates large overshoots and the convergence to the target position is very slow. The same observation can be noticed with the classical RISENN. Significant overshoots are generated with a larger $5 \%$ settling time. In addition, the evaluation of the function $E$ (32) shows that the PD consumes much more control energy. This is visible through the saturation of its control input which reflects the high driving force and damping required to displace the head, whereas the one of the RISE-NN reacts more smoothly and does not reach the maximum limits.

Fig. 5(a $\left.a_{3}\right)$ shows that until $+80 \%$ of uncertainty, the effect on the HDD closed-loop behavior is negligible: the system ensures faster convergence, smaller tracking errors and oscillations compared to those of the PD and the RISENN controllers. The effectiveness of P-RISE-NN is due to the anticipatory behavior. Hence, the prediction process predicts the system evolution over the predefined horizon $N_{p}$ and choose the best value of the feedback gain. This generates the optimal gain $k_{s}^{o p t}$ to have the best possible tracking performances. 
(a)

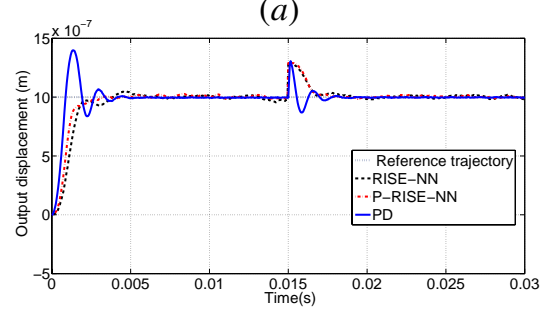

(b)

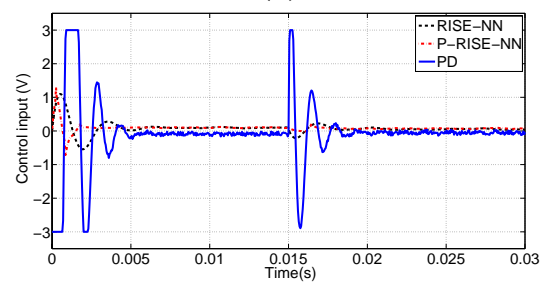

(c)

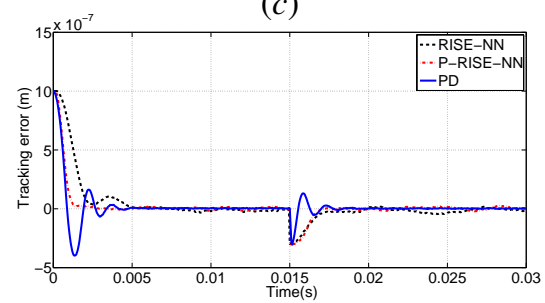

Fig. 4: Nominal case with disturbance rejection (Plots with PD, RISE-NN and P-RISE-NN controllers): (a) Evolution of the measured outputs $(b)$ Evolution of the control input.
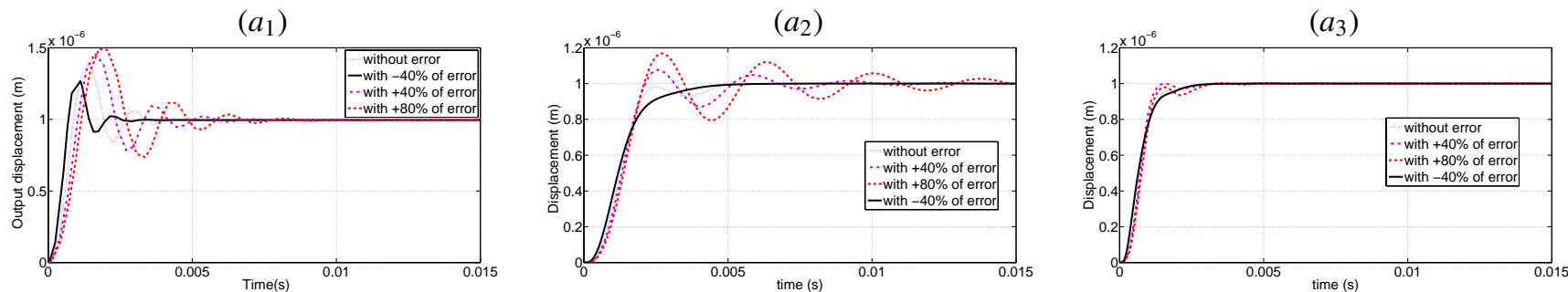

Fig. 5: Robustness towards parameter' uncertainties (Evolution of the measured outputs): Plots with $\left(a_{1}\right)$ PD controller, $\left(a_{2}\right)$ RISE-NN controller and $\left(a_{3}\right)$ P-RISE-NN controller.

$\left(b_{1}\right)$

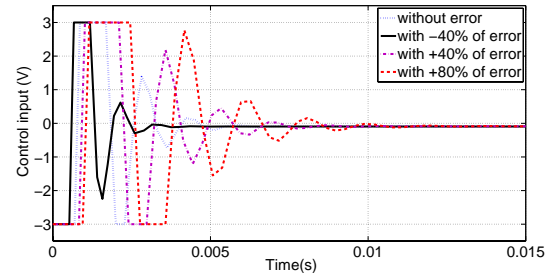

$\left(b_{2}\right)$

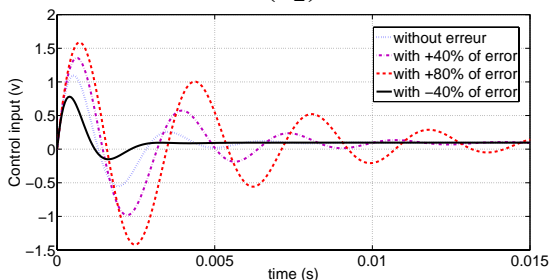

$\left(b_{3}\right)$

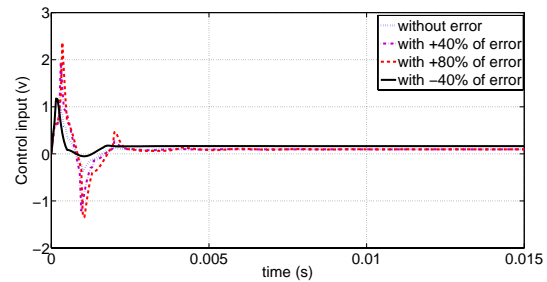

Fig. 6: Robustness towards parameter' uncertainties (Evolution of the control input): Plots with $\left(b_{1}\right)$ PD controller, $\left(b_{2}\right)$ RISE-NN controller and $\left(b_{3}\right)$ P-RISE-NN controller.

\section{CONCLUSIONS AND FUTURE WORK}

In this paper, a prediction-based optimal gain selection approach has been presented for RISE based NN control applied to a HDD servo positioning system. Through numerical simulations, it has been shown that the proposed new controller P-RISE-NN is able to achieve a quick displacement as well as an accurate positioning of the R/W head tip at the desired target. Indeed, the tracking error is minimized and converges rapidly to near zero. It is worth noting that the predictive behavior of the P-RISE-NN technique guarantees a better rejection of external disturbances as well as robustness against parametric uncertainties compared to both PD and classical RISE-NN controllers. Our future work involves the real-time implementation of this novel control solution on a HDD.

\section{REFERENCES}

[1] B. Xian, D. Dawson, M. De Queiroz, and J. Chen, "A continuous asymptotic tracking control strategy for uncertain nonlinear systems," IEEE Tran. on Automatic and Control, vol. 49, no. 7, pp. 1206-1211, July 2004.

[2] Z. Gay, M. De Queiroz, and D. Dawson, "Robust adaptive asymptotic tracking of nonlinear systems with additive disturbance," IEEE Trans. on Automatic and Control, vol. 51, no. 3, pp. 524-529, 2006.

[3] X. Zhang, A. Behal, D. M. Dawson, and B. Xian, "Output feedback control for a class of uncertain mimo nonlinear systems with nonsymmetric input gain matrix," in IEEE Conference on Decision and
Control, the European Control Conference, Spain, 2005, pp. 77627767.

[4] S. Gupta, D. Aiken, G. Hu, and W. Dixon, "Lyapunov-based range and motion identification for a nonaffine perspective dynamic system," in IEEE American Control Conference, Minneapolis, MN, 2006, pp. 4471-4476.

[5] W. Dixon, Y. Fang, D. Dawson, and T. Flynn, "Range identification for perspective vision systems," IEEE Trans. on Automatic and Control, vol. 48 , no. 12 , pp. 2232-2238, December 2003.

[6] C. Makkar, G. Hu, W. Sawyer, and W. Dixon, "Lyapunov-based tracking control in the presence of uncertain nonlinear parameterizable friction," IEEE Trans. on Automatic and Control, vol. 52, no. 10, pp. 1988-1994, October 2007.

[7] S. Ge, C. Hang, and L. Woon, "Adaptive neural network of robot manipulators in task space," IEEE Trans. on Industrial Electronics, vol. 44, no. 6, pp. 746-752, 1997.

[8] B. Chen, K. Lee, T.H.and Peng, and V. Venkataramanan, Hard Disc Drive Servo-Systems. Springer, 2006.

[9] P. San, B. Ren, S. Ge, T. Lee, and J. Liu, "Adaptive neural network control of hard disk drives with hysteresis friction nonlinearity," IEEE Trans.s on Control Systems Technology, vol. 19, no. 2, pp. 351-358, March 2011.

[10] X. Liu and J. Liu, "Analysis and measurement of torque hysteresis of pivot bearing in hard disk drive," Tribology International, vol. 32, pp. 125-130, March 1999.

[11] F. Lewis, "Nonlinear network structures for feedback control," Asian Journal of Control, vol. 1, no. 4, pp. 205-228, 1999.

[12] P. M. Patre, W. MacKunis, K. Kent, and W. Dixon, "Asymptotic tracking for uncertain dynamic systems via a multilayer neural network feedforward and rise feedback control structure," IEEE Trans. on Automatic Control, vol. 53, no. 9, pp. 2180-2185, 2008.

[13] E. Camacho and C. Bordons, Model Predictive Control. Springer, 2004. 